\title{
SMC-Based Synchronization of Multiple Inertial Measurement Units with Application to Attitude Tracking Control
}

\author{
Yuping He $(\mathbb{D})$ and Shijie Zhang $(D)$ \\ Research Center of Satellite Technology, Harbin Institute of Technology, Harbin 150080, China \\ Correspondence should be addressed to Yuping He; 13b918053@hit.edu.cn
}

Received 26 October 2020; Revised 30 December 2020; Accepted 25 January 2021; Published 17 February 2021

Academic Editor: Shihong Ding

Copyright (c) 2021 Yuping He and Shijie Zhang. This is an open access article distributed under the Creative Commons Attribution License, which permits unrestricted use, distribution, and reproduction in any medium, provided the original work is properly cited.

In this paper, the attitude control of aircraft with multiple inertial measurement units under the influence of unknown gyro zero drift and external disturbance is studied. First of all, the observers are designed to estimate the zero drift biases based on the consensus algorithm. The angular velocity used for aircraft control is obtained by compensating the biases. Then, considering the external disturbance in the aircraft motion, this paper introduces a super-twisting sliding-mode algorithm to design the observer in order to compensate the disturbance. In addition, based on the proposed observers, a controller is designed to realize attitude control of the aircraft with the gyro zero drift and the external disturbance. Finally, the simulation results are given to verify the effectiveness of the proposed control law.

\section{Introduction}

The aircraft is the unmanned robot operated by radio remote control equipment or self-contained program control device. It is widely used in transportation, reconnaissance, photography, and other fields [1]. In recent years, the research on the aircraft is hot at home and abroad because of many advantages of the aircraft, such as the easy maintenance, high maneuverability, and high reliability [2]. In this paper, the hot spot field of aircraft-attitude control is studied. It is worth mentioning that there are two main factors that interfere with the attitude control of the aircraft: external disturbance of the aircraft and measurement deviation of the inertial measurement unit (IMU). The external disturbance of the aircraft, such as the disturbance of air flow, will lead to the unstable movement of the aircraft and lead to the failure of control. Measurement deviations, such as zero drift biases, can lead to inaccurate angular velocity of the aircraft, resulting in aircraft control failure. In addition, the attitude information obtained by a single IMU cannot meet the requirements of attitude control.

For the external disturbance, most of the current research results use the observer to estimate the disturbance and compensate the controller. As a class of nonlinear observers, sliding mode observers can work under less conservative conditions [3, 4]. Mofid et al. [5] proposed an adaptive sliding mode disturbance observer for synchronization of a fractional-order Dadras-Momeni chaotic system with time-varying disturbances is presented. Hou et al. [6] proposed a novel disturbance observer with the supertwisting sliding mode technique in order to improve the performance of the permanent magnet synchronous motor speed regulation system. To deal with the effect of the angular velocity biases caused by the zero drift, there are two main solutions in the current literature: (1) through the physical calibration of the gyro to solve the effect of zero drift bias on the measurement results [7-10] and (2) the zero drift biases can be estimated and compensated by attitude estimation [11-13], which is like the idea of disturbance estimation and compensation [14]. The authors in [15] proposed a novel analytic calibration of gyro biases with arbitrary double position based on the transformation model of gyro biases. According to the analytic-coarse-alignment principle, the sensitivities of Euler angles with respect to inertial sensor biases are analyzed uniquely. The authors in [16] proposed a bias compensation and parameter 
calibration method based on Gauss-Newton algorithm. The method can effectively suppress zero drift, but its operation is cumbersome. Unscented Kalman filters (UKF) [17], extended Kalman filters (EKF) [18], and nonlinear complementary filters [19] are the popular methods of attitude estimation in the literature. However, small aircrafts are often equipped with low-precision gyros, which often carry non-Gaussian noise during the measurement process. This will cause the Kalman estimation algorithm to fail. The authors in [20] proposed a solution by constructing an observer. This work designed an observer for estimating the constant biases caused by the zero drift and proposed a coupled nonlinear spacecraft attitude controller. The authors in [21] presented a nonlinear attitude estimator based on an alternate error function, and this estimator can be implemented using a low-cost inertial measurement unit.

The conventional IMU consists of an accelerometer and a gyro for aircraft attitude control. IMU has also been widely used in smart wearable devices [22], navigation of unmanned systems [23, 24], guidance of military equipment [25], and so forth. Due to the increasing demand for inertial measurement devices in various fields, higher requirements have been placed on the measurement accuracy of inertial devices. In recent years, researchers have also proposed the configuration design of multiple accelerometers and gyro arrays. The authors in [26] analyzed the performance of the IMU and its various sensor configurations. The measurement accuracy is effectively improved by selecting the appropriate geometric configuration and the combination of multiple inertial navigation sensors. The authors in [27] used a single-axis gyro and multiple spatially distributed accelerometers to achieve the correction of the attitude measurement value. The authors in [28] designed three two-axis accelerometer inertial measurement units to measure the angular velocity of six degrees of freedom and compensated the measurement error through data fusion. The authors in [29] studied 12 accelerometers and gyros assigned IMU and proposed an EKF program to estimate the direction and amplitude of angular velocity. Research shows that attitude measurement systems that use multiple sensors have advantages over single sensor. The main difficulty in attitude control of multiple IMUs is how to design the multiple observers to synchronize [30-32]. Also, there some results have been reported about the synchronization of multiple observers/controllers [33-35].

So far, most of the research results on attitude control adopt single IMU. For the large aircraft, a single IMU can no longer meet its technical requirements [36]. Since the measurement range of a single gyro is easily saturated in high dynamic motion, its application is also limited. In addition, the attitude control of the aircraft will be unstable due to the zero drift and external disturbance. Therefore, considering the above problems, it is necessary to study the anti-interference control of aircraft attitude under multiple IMUs.

Motivated by the above discussions, this paper studies the attitude tracking control law of the aircraft with zero drift and external disturbance based on multiple inertial measurement units. As in the work [37], this paper assumes that the biases caused by the zero drift are constant in this paper. In order to solve this problem, this article will make full use of the data measured by each IMU to design the nonlinear observer, estimate the zero drift biases, and compensate for it. Then, a super-twisting sliding mode observer will be designed to compensate the disturbance. In addition, a tracking controller will be proposed. By constructing a suitable Lyapunov function, the asymptotic stability of the attitude system will be strictly proven. The asymptotic stability of the combined system of the observer and controller will be proved by the theory of cascade system. Finally, a simulation will be given to verify the effectiveness of the proposed control law. The main innovation points/ contributions of the method proposed in this paper are as follows. (1) In this paper, multiple IMUs are used for measurement and observers are designed based on consensus algorithm. The observer designed in this paper can estimate the zero drift biases. (2) In this paper, a supertwisting sliding mode observer is used to estimate and eliminate external disturbances. Combined with the proposed attitude observer, the internal disturbance and external disturbance can be eliminated. (3) The aircraft model has highly nonlinear and strong coupling, the design of the control system is very difficult. In this paper, by analyzing the aircraft attitude system, the attitude observer and controller are proposed. By compensating for the zero drift, the attitude tracking control of the aircraft is realized.

\section{Preliminaries}

2.1. Problem Description. The attitude model is mainly composed of kinematics and dynamics equations. In this article, the Euler angles will be used to describe the attitude with respect to the inertial frame. The Euler vector is given by

$$
\Phi=[\phi, \theta, \psi]^{T} \in \mathbb{R}^{3},
$$

where $\phi$ is the angle of pitch, $\theta$ is the angle of roll, and $\psi$ is the yaw angle.

Based on the Euler angles, the attitude kinematics equation of the aircraft is

$$
\dot{\Phi}=W(\Phi) w
$$

where $w \in \mathbb{R}^{3}$ is the angular velocity which can be measured by gyro, and the matrix $W(\Phi)$ is given by

$$
W(\Phi)=\left[\begin{array}{ccc}
1 & \sin \phi \tan \theta & \cos \phi \tan \theta \\
0 & \cos \phi & -\sin \phi \\
0 & \sin \phi \sec \theta & \cos \phi \sec \theta
\end{array}\right] .
$$

In fact, the inertial measurement unit composed of a single gyro and a single accelerometer cannot meet the measurement accuracy requirements of the aircraft. To this end, this paper will adopt multiple IMUs to attitude control.

In this paper, $n$ sets of IMUs are considered for measurement and let $\Gamma=\{1,2, \ldots, n\}$. Each IMU is independent, and its measurement results include the angular velocity and the attitude, i.e., $m_{i}=\left[w_{g i}, \Phi_{i}\right]^{T}$. In addition, due to the inherent shortcomings of the device, the gyro will produce 
zero drift, which leads to the measurement of angular velocity with unknown bias, as shown in [20]. The measured angular velocity of $i$ th IMU can be written as follows:

$$
w_{g i}=\left[w_{g, 1}, w_{g, 2}, w_{g, 3}\right]^{T}=w+b_{i},
$$

where $b_{i}$ is the unknown gyro bias of $i$ th IMU's gyro.

The control objective of this paper is to obtain accurate attitude data through multisensor measurement data. On this basis, the attitude tracking control of the aircraft is carried out.

Assumption 1 (see [37]). This paper assumes that the zero drift bias $b_{i}$ of $i$ th IMU's gyro is a constant value, and the zero drift bias of each gyro is different.

\subsection{Related Definition and Lemma}

Definition 1. For any $\beta>0$, the nonlinear function $\operatorname{sig}^{\beta}(x)$ is defined as

$$
\operatorname{sig}^{\beta}(x)=\operatorname{sign}(x)|x|^{\beta} .
$$

Lemma 1 (super-twisting sliding-mode algorithm, see [38]). Consider the system

$$
\begin{aligned}
& \dot{\tilde{x}}_{1}=-\iota_{1} \operatorname{sig}^{1 / 2}\left(\tilde{x}_{1}\right)+\iota_{2} \tilde{x}_{2}, \\
& \dot{\tilde{x}}_{2}=-l_{3} \operatorname{sign}\left(\tilde{x}_{1}\right)+v\left(\tilde{x}_{1}, \tilde{x}_{2}, t\right),
\end{aligned}
$$

where $\iota_{i}>0$ are the gains to be designed and $v\left(\tilde{x}_{1}, \tilde{x}_{2}, t\right)$ are the perturbation terms; it satisfies

$$
\left|v\left(\tilde{x}_{1}, \tilde{x}_{2}, t\right)\right| \leq \delta
$$

where $\delta>0$. Under some conditions on $\iota_{1}, \iota_{2}, \iota_{3}$, the equilibrium point of system (6) will converge to the origin in a finite time $T$.

2.3. Graph Theory. In this paper, the multiple IMU will be considered. Each IMU can be seen as a node. The information interaction among $n$ nodes can be represented by the directed graph $G(A)=\{V, E, A\} . V=\left\{v_{i}, i=1, \ldots, n\right\}$ is the set of nodes, $E \subseteq V \times V$ is the set of edges, and $A=\left[a_{i j}\right] \in R^{n \times n}$ is the weighted adjacency matrix of the graph $G(A)$ with nonnegative adjacency elements $a_{i j}$. If there is an edge from node $j$ to node $i$, i.e., $\left(v_{j}, v_{i}\right) \in E$, then $a_{i j}>0$, which means there exists an available information channel from node $j$ to node $i$. The set of neighbors of node $i$ is denoted by $N_{i}=\left\{j:\left(v_{j}, v_{i}\right) \in E\right\}$. The out-degree of node $v_{i}$ is defined as $\operatorname{deg}_{\text {out }}\left(v_{i}\right)=d_{i}=\sum_{j=1}^{n} a_{i j}=\sum_{j \in N_{i}} a_{i j}$. Then, the degree matrix of digraph $G$ is $D=\operatorname{diag}\left\{d_{1}, \ldots, d_{n}\right\}$ and the Laplacian matrix of digraph $G$ is $L=D-A$.
A path in graph $G$ from $v_{i_{1}}$ to $v_{i_{k}}$ is a sequence of $v_{i_{1}}, v_{i_{2}}, \ldots, v_{i_{k}}$ of finite nodes starting with $v_{i_{1}}$ and ending with $v_{i_{k}}$ such that $\left(v_{i}, v_{i_{l+1}}\right) \in E$ for $l=1,2, \ldots, k-1$. The graph $G$ is connected if there is a path between any two distinct vertices.

\section{Main Results}

To solve the attitude tracking maneuver in the presence of unknown gyro bias, the main design procedure is divided into two steps:

The observer for each IMU to estimate the gyro bias is designed based on the consensus algorithm

An attitude controller based on the observer is designed to realize asymptotically stable attitude tracking

3.1. Design of Nonlinear Observers Based on Consensus Algorithm. Because of the nonlinear structure of attitude system (2), it is difficult to design an observer under multiple IMUs. In this paper, let $\widehat{b}_{i}=\left[\widehat{b}_{i, 1}, \widehat{b}_{i, 2}, \widehat{b}_{i, 3}\right]^{T}$ denote the estimation of the gyro unknown bias for the $i$ th IMU's gyro and $\widehat{\Phi}_{i}$ denote the estimated value of the real-time attitude of the aircraft for the $i$ th IMU's accelerometer. Based on models (2) and (4), we can design the following observer.

Theorem 1. Considering the attitude system (2) and Assumption 1, if the observers are designed as

$$
\begin{aligned}
& \dot{\widehat{\Phi}}_{i}=W\left(\Phi_{i}\right)\left(w_{g i}-\widehat{b}_{i}\right)-k_{1} e_{i}-\sum_{j \in N_{i}} a_{i j}\left(e_{i}-e_{j}\right), \\
& \dot{\hat{b}}_{i}=k_{2} W^{T}\left(\Phi_{i}\right) e_{i}, \quad i \in \Gamma,
\end{aligned}
$$

where $k_{1}>0, k_{2}>0$, and $e_{i}=\left[e_{i, 1}, e_{i, 2}, e_{i, 3}\right]^{T}=\widehat{\Phi}_{i}-\Phi_{i}$, then the estimation value $\left(\widehat{\Phi}_{i}, \widehat{b}_{i}\right)$ will globally asymptotically converge to true value $\left(\Phi_{i}, b_{i}\right)$.

Proof. First of all, let the estimation error for gyro bias be $f_{i}=\widehat{b}_{i}-b$. Then, the error dynamical equation can be obtained:

$$
\begin{aligned}
& \dot{e}_{i}=-W\left(\Phi_{i}\right) f_{i}-k_{1} e_{i}-\sum_{j \in N_{i}} a_{i j}\left(e_{i}-e_{j}\right), \\
& \dot{f}_{i}=k_{2} W^{T}\left(\Phi_{i}\right) e_{i}, \quad i \in \Gamma
\end{aligned}
$$

Choose the Lyapunov function for the error system (9) as

$$
V=\sum_{i=1}^{n}\left[\frac{k_{2}}{2} \sum_{k=1}^{3} e_{i, k}^{2}+\frac{1}{2} f_{i}^{T} f_{i}\right],
$$

whose derivative is 


$$
\begin{aligned}
\dot{V} & =\sum_{i=1}^{n}\left[k_{2} \sum_{k=1}^{3} \dot{e}_{i, k} e_{i, k}+\dot{f}_{i}^{T} f_{i}\right] \\
& =\sum_{i=1}^{n}\left[k_{2} e_{i}^{T}\left[-W\left(\Phi_{i}\right) f_{i}-k_{1} e_{i}-\sum_{j=1}^{n} a_{i j}\left(e_{i}-e_{j}\right)\right]+k_{2}\left[W^{T}\left(\Phi_{i}\right) e_{i}\right]^{T} f_{i}\right] \\
& =-k_{1} k_{2} \sum_{i=1}^{n} e_{i}^{T} e_{i}-k_{2} \sum_{i=1}^{n} e_{i}^{T} \sum_{j=1}^{n} a_{i j}\left(e_{i}-e_{j}\right) \\
& =-k_{1} k_{2} \sum_{i=1}^{n} e_{i}^{T} e_{i}-\frac{k_{2}}{2} \sum_{i=1}^{n} \sum_{j=1}^{n} a_{i j}\left(e_{i}-e_{j}\right)^{T}\left(e_{i}-e_{j}\right) \\
& =-k_{1} k_{2} \sum_{i=1}^{n} \sum_{k=3}^{n} e_{i, k}^{2}-\frac{k_{2}}{2} \sum_{i=1}^{n} \sum_{j=1}^{n} a_{i j} \sum_{k=1}^{3}\left(e_{i, k}-e_{j, k}\right)^{2} \leq 0 .
\end{aligned}
$$

Based on LaSalle's invariant principle, it is easy to get the conclusion that $\left(e_{i}, f_{i}\right)$ will globally asymptotically converge to the origin, i.e., $\left(\widehat{\Phi}_{i}, \widehat{b}_{i}\right)$ will globally asymptotically converge to true value $\left(\Phi_{i}, b_{i}\right)$. The proof is completed.

Remark 1. With observer (8), the gyro of $i$ th IMU can estimate the true value of the gyro zero drift $\widehat{b}_{i}$. By compensating the bias, the angular velocity of the $i$ th IMU can be obtained, i.e., $\bar{w}_{i}=w_{g i}-\widehat{b}_{i}$. The angular velocity used to control the attitude of the aircraft in this paper is given by

$$
\bar{w}=\frac{1}{n} \sum_{i=1}^{n}\left(w_{g i}-\widehat{b}_{i}\right) .
$$

3.2. Design of a Nonlinear Controller Based on a Super-Twist Observer. The dynamic equation of the aircraft is as follows:

$$
J \dot{w}=-w^{\times} J w+\tau+d,
$$

where $J \in \mathbb{R}^{3}$ and $\tau \in \mathbb{R}^{3}$ are, respectively, the positive definite inertia matrix, and the control torque of spacecraft, $d$ denotes the external disturbance satisfies $d \leq d^{*}$ with a positive constant $d^{*}$, and the symbol $(\cdot)^{\times}$denotes the skew symmetry matrix, i.e.,

$$
x^{\times}=\left[\begin{array}{ccc}
0 & -x_{3} & x_{2} \\
x_{3} & 0 & -x_{1} \\
-x_{2} & x_{1} & 0
\end{array}\right], \quad \forall x \in \mathbb{R}^{3} .
$$

The desired attitude is described as $\Phi_{d}=\left[\phi_{d}, \theta_{d}, \psi_{d}\right]^{T}$, and it is constant in this paper. Define the attitude error between the true value and the desired value as $\Phi_{e}=\left[\Phi_{e 1}, \Phi_{e 2}, \Phi_{e 3}\right]^{T}=\Phi-\Phi_{d}$. To sum up, the attitude error system of the aircraft is

$$
\begin{aligned}
& \dot{\Phi}_{e}=\dot{\Phi}-\dot{\Phi}_{d}=W(\Phi) w, \\
& J \dot{w}=-w^{\times} J w+\tau+d .
\end{aligned}
$$

Theorem 2. For the attitude error system (15), if the controller is designed as

$$
\begin{aligned}
\tau & =-k_{3} \bar{w}-k_{4} W^{T}(\Phi) \Phi_{e}-\widehat{d}, \\
\dot{\hat{w}} & =J^{-1}\left(-\bar{w}^{\times} J \bar{w}-k_{3} \bar{w}-k_{4} W^{T}(\Phi) \Phi_{e}\right)-k_{5} \operatorname{sig}^{1 / 2}(\widehat{w}-\bar{w}), \\
\dot{\hat{d}} & =-k_{6} \operatorname{sign}(\widehat{w}-\bar{w}),
\end{aligned}
$$

where $k_{3}, k_{4}, k_{5}, k_{6}>0, \widehat{w}$ represents the estimate of the angular velocity, and $\hat{d}$ denotes the estimate of the external disturbance; then, the asymptotic tracking of attitude can be implemented.

Proof. It can be known from Theorem 1 that $\bar{w}$ will eventually converge to $w$. The observation errors $e_{1}$ and $e_{2}$ are defined as

$$
\begin{aligned}
& e_{1}=\widehat{w}-\bar{w}, \\
& e_{2}=\widehat{d}-d,
\end{aligned}
$$

whose derivative is

$$
\begin{aligned}
& \dot{e}_{1}=-k_{5} \operatorname{sig}^{1 / 2}\left(e_{1}\right)+J^{-1} e_{2}, \\
& \dot{e}_{2}=-k_{6} \operatorname{sign}\left(e_{1}\right) .
\end{aligned}
$$

From Lemma 1 , we know that $e_{1}$ and $e_{2}$ will converge to 0 in a finite time. In other words, the estimated disturbance value $\widehat{d}$ will converge to the true value $d$ in a finite time $T_{1}$. After $T_{1}$, it follows (15) and (16) that

$$
\begin{aligned}
& \dot{\Phi}_{e}=W(\Phi) w \\
& J \dot{w}=-w^{\times} J w-k_{3} \bar{w}-k_{4} W^{T}(\Phi) \Phi_{e} .
\end{aligned}
$$

It follows (9), (15), and (19) that 


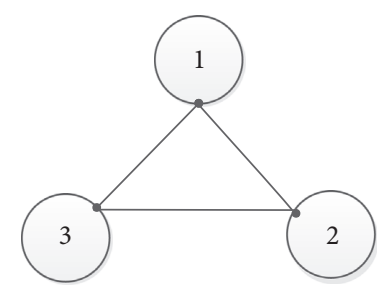

FIgURE 1: The information exchange of three IMUs.

$$
\begin{aligned}
\dot{\Phi}_{e} & =W(\Phi) w, \\
J \dot{w} & =-w^{\times} J w+u_{1}+\left(u_{2}-u_{1}\right), \\
\dot{e}_{i} & =-W\left(\Phi_{i}\right) f_{i}-k_{1} e_{i}-\sum_{j \in N_{i}} a_{i j}\left(e_{i}-e_{j}\right), \\
\dot{f}_{i} & =k_{2} W^{T}\left(\Phi_{i}\right) e_{i}, \quad i \in \Gamma,
\end{aligned}
$$

where $u_{1}=-k_{3} w-k_{4} W^{T}(\Phi) \Phi_{e}$ and $u_{2}=-k_{3} \bar{w}-k_{4} W^{T}(\Phi)$ $\Phi_{e}$. Obviously, system (20) can be regarded as a cascaded system with the cascaded term $\left(u_{1}-u_{2}\right)$. Next, we will use cascaded systems' theory [39] to analyze the stability of system (20). The proof includes two steps:

Step 1: proof of global asymptotic stability of subsystems.

Consider the following system:

$$
\begin{aligned}
& \dot{\Phi}_{e}=W(\Phi) w \\
& J \dot{w}=-w^{\times} J w+u_{1} .
\end{aligned}
$$

Choose the following Lyapunov function for the attitude error system (21):

$$
H=\frac{k_{4}}{2} \sum_{j=1}^{3} \Phi_{e j}^{2}+\frac{1}{2} w^{T} J w
$$

Based on the matrix $w^{T} w^{\times} J w$ which is skew symmetric, the derivative of $H$ along system (21) is

$$
\begin{aligned}
\dot{H} & =k_{4} \sum_{j=1}^{3} \Phi_{e j} \dot{\Phi}_{e j}+w^{T} J \dot{w} \\
& =k_{4} w^{T} W^{T}(\Phi) \Phi_{e}+w^{T}\left(-w^{\times} J w+u_{1}\right) \\
& =k_{4} w^{T} W^{T}(\Phi) \Phi_{e}+w^{T}\left(-k_{3} w-k_{4} W^{T}(\Phi) \Phi_{e}\right) \\
& =-k_{3} w^{2} \leq 0
\end{aligned}
$$

Based on LaSalle's invariant principle, it can be concluded that $\dot{H}(t) \longrightarrow 0$ as $t \longrightarrow \infty$, which implies that system (21) is globally asymptotically stable. It is easy to get the conclusion that $\left(\Phi_{e}, w\right) \longrightarrow 0$ as $t \longrightarrow \infty$.

Clearly, system (9) and system (21) are globally asymptotically stable with equilibrium $\left(w, \Phi_{e}, e_{i}\right.$, $\left.f_{i}\right)=(0,0,0,0)$.

Step 2: proof of global state bounded of cascaded system.
According to the cascaded systems' theory [39], cascades of the globally asymptotically stable systems remain globally asymptotically stable if and only if solutions are globally bounded. With these facts in mind, to prove the stability of system (20), we only need to prove that the state of system (20) is globally bounded. To achieve this objective, choose the Lyapunov function:

$$
U=V+H .
$$

According to (11) and (23), it obtains

$$
\begin{aligned}
\dot{U}= & -k_{1} k_{2} \sum_{i=1}^{n} e_{i}^{2}-\frac{k_{2}}{2} \sum_{i=1}^{n} \sum_{j=1}^{n} a_{i j}\left(e_{i}-e_{j}\right)^{2}-k_{3} w^{2} \\
& +w^{T}\left(u_{1}-u_{2}\right) .
\end{aligned}
$$

Since systems (9) and (21) are globally asymptotically stable, $f_{i}$ and $w$ are globally bounded. There is a constant $0<d<+\infty$ such that $\left\|u_{1}-u_{2}\right\| \leq d$. We have

$$
\dot{U} \leq-k_{1} k_{2} \sum_{i=1}^{n} e_{i}^{2}-\frac{k_{2}}{2} \sum_{i=1}^{n} \sum_{j=1}^{n} a_{i j}\left(e_{i}-e_{j}\right)^{2}-k_{3} w^{2}+\sum_{j=1}^{3} w_{i} d .
$$

Clearly, when $k_{1} k_{2} \sum_{i=1}^{n} e_{i}^{2}+\left(k_{2} / 2\right) \sum_{i=1}^{n} \sum_{j=1}^{n} a_{i j} \quad\left(e_{i}\right.$ $\left.-e_{j}\right)^{2}+k_{3} w^{2}>\sum_{j=1}^{3} w_{i} d$, then $\dot{U}<0$. Thus, the state $\left(w, \Phi_{e}, e_{i}, f_{i}\right)$ is globally bounded.

With this fact in mind, it can be concluded that the state of system (20) is globally bounded, which implies that this system is asymptotically stable. That is to say the attitude tracking is achieved asymptotically.

Remark 2. According to the previous proof, when the observer gains meet $k_{1}, k_{2}>0$ and the controller gains meet $k_{3}, k_{4}, k_{5}, k_{6}>0$, the attitude asymptotic stability tracking can be realized. In practical applications, the observer gain and controller gain can be selected step by step to obtain better performance.

\section{Simulation Example}

A simulation example is given to verify the effectiveness of the proposed method. In this paper, three inertial measurement units are selected for simulation. Next, the initial values, parameters, and real values required for simulation in this paper are given. The information exchange topology among IMU is shown in Figure 1. The initial values of the observer part are selected as $\widehat{b}_{i}(0)=[0.1,0.1,0.1]^{T} \mathrm{rad} / \mathrm{sec}$ and $\widehat{\Phi}_{i}(0)=$ $[0.3,0.3,0.3]^{T} \mathrm{deg}, i=1,2,3$. The observer parameters are 

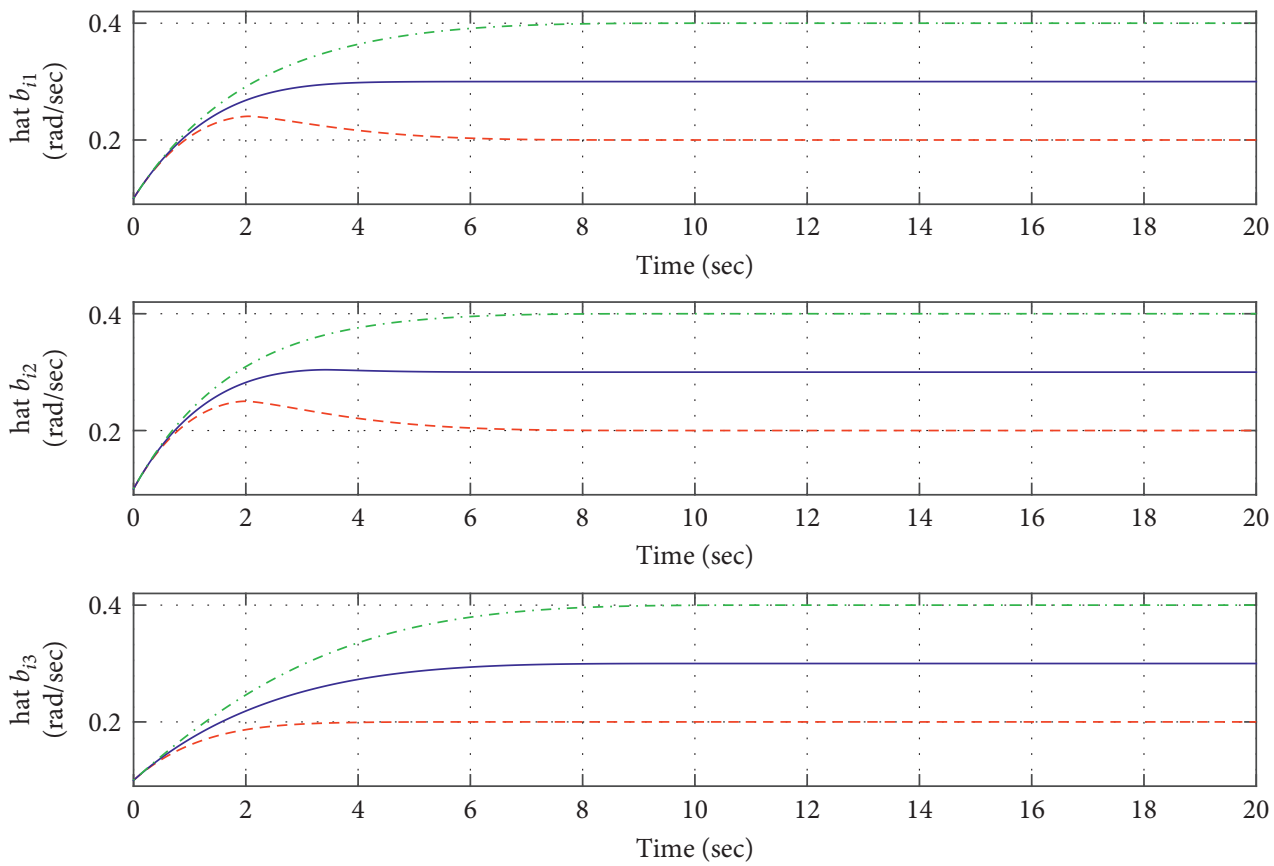

-. - IMU

$-\mathrm{IMU}_{2}$

-.- $\mathrm{IMU}_{3}$

FIgURE 2: The bias estimation $\widehat{b}_{i}$ of $i$ th IMU by using the proposed observer.
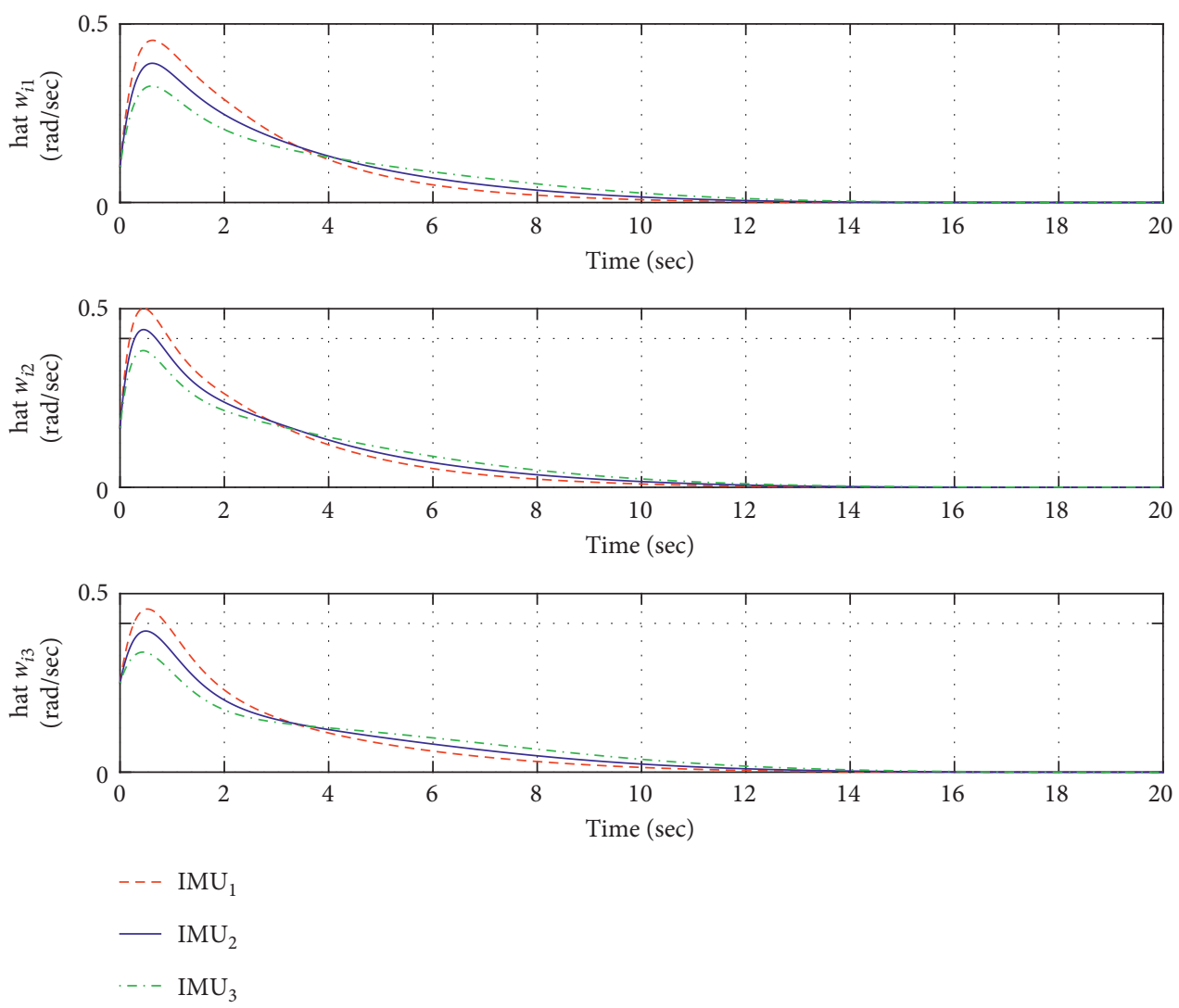

FIgURE 3: The angular velocity $\widehat{w}_{i}$ of $i$ th by using the proposed observer. 

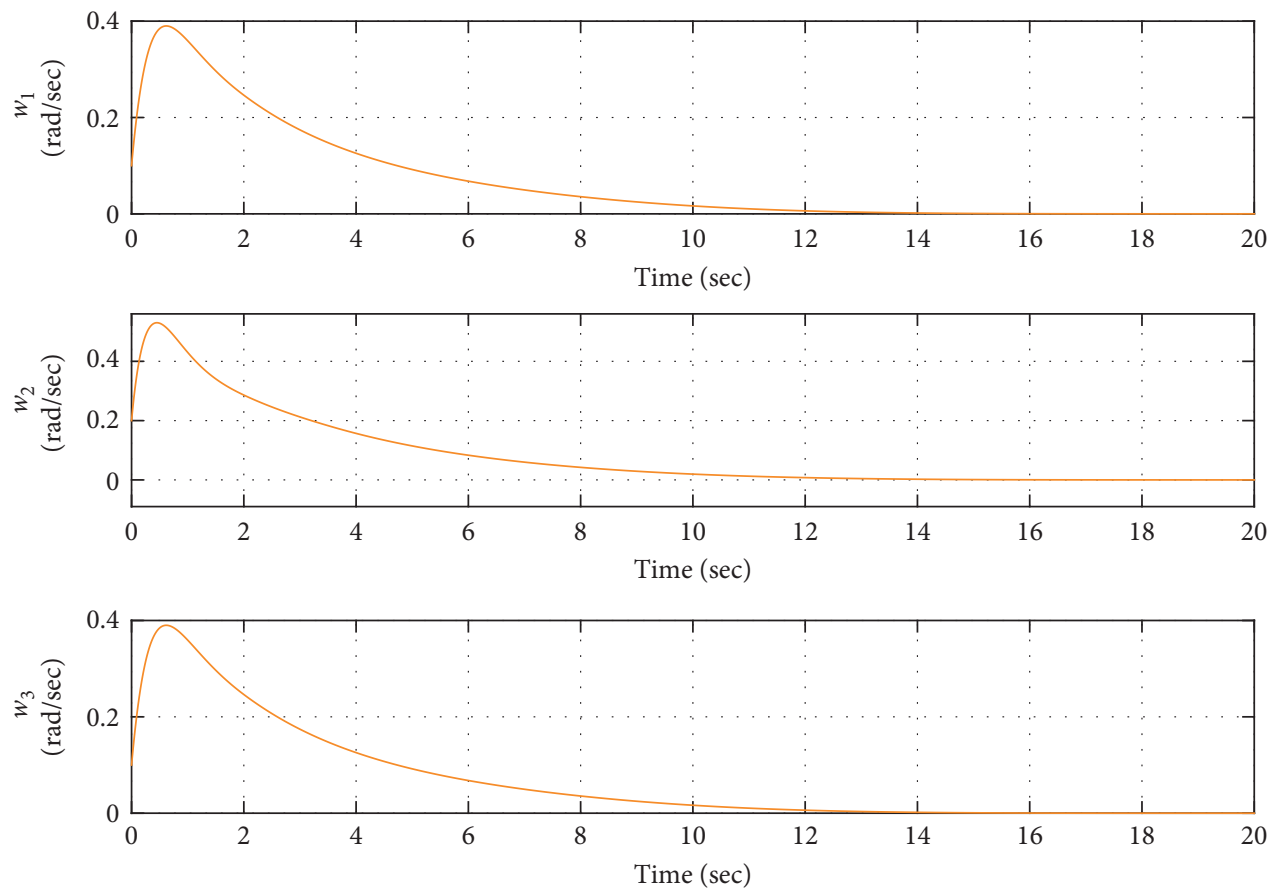

FIgURE 4: The response curve of aircraft's angular velocity.
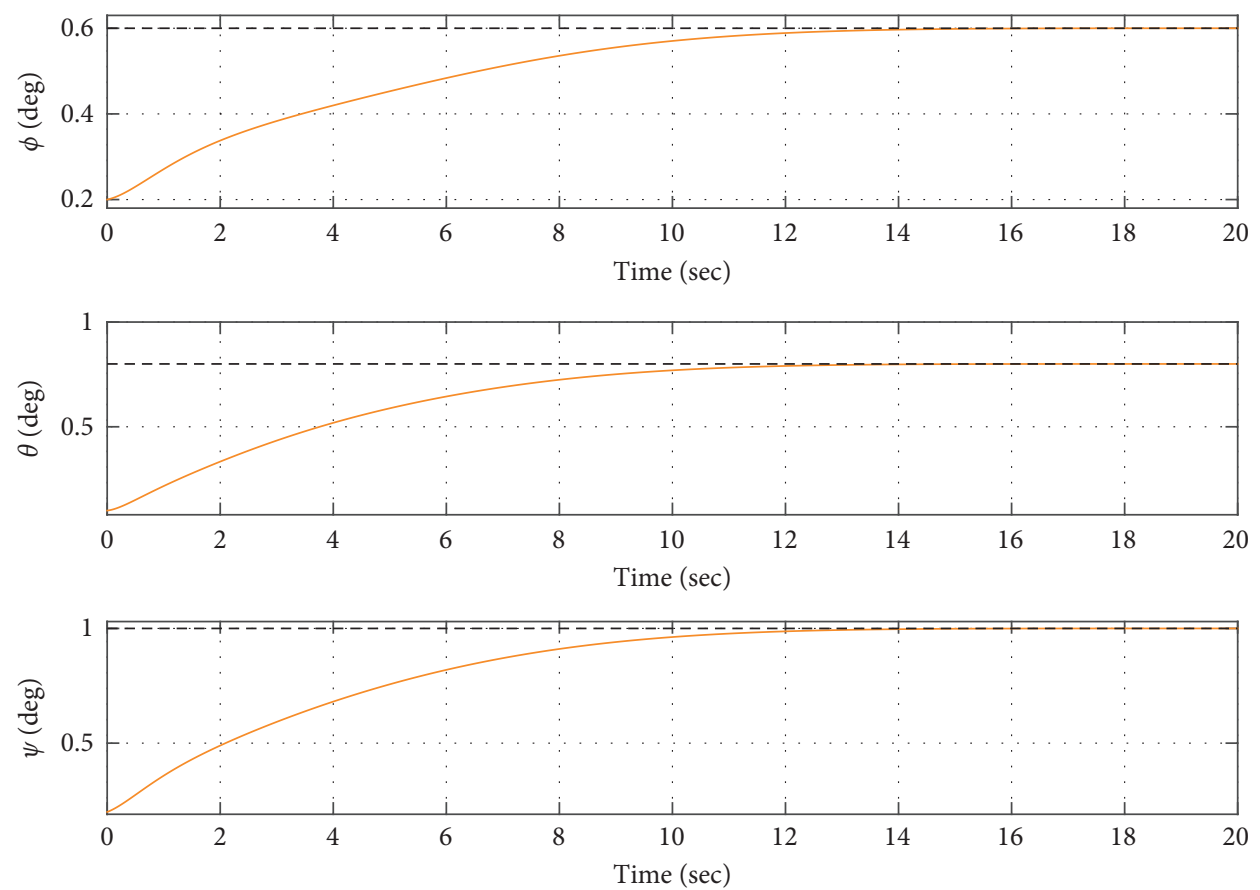

FIGURE 5: The response curve of aircraft's attitude.

$k_{1}=13$ and $k_{2}=2.36$. The initial values of the controller are selected as $\Phi(0)=[0.2,0.1,0.2]^{T}$ deg and $w(0)=[0.1,0.2$, $0.3]^{T} \mathrm{rad} / \mathrm{sec}$. The controller parameters are $k_{3}=9.754$, $k_{4}=3.21, k_{5}=2.3$, and $k_{6}=1.4$. The desired attitude of the aircraft is set as $\Phi_{d}=[0.6,0.8,1]^{T}$ deg. The true biases of the
IMUs' gyro are selected as $b_{\text {turel }}=[0.2,0.2,0.2]^{T} \mathrm{rad} / \mathrm{sec}$, $b_{\text {ture2 }}=[0.3,0.3,0.3]^{T} \mathrm{rad} / \mathrm{sec}$, and $b_{\text {ture3 }}=[0.4,0.4,0.4]^{T} \mathrm{rad} /$ sec. The external disturbance is set as $d(t)=$ $[0.2+\sin (t), 0.3+\sin (3 t), 0.1+\sin (t)]^{T}$. The inertia matrix of the aircraft is chosen as that in [40]: 


$$
J=\left[\begin{array}{ccc}
1 & 0 & 0 \\
0 & 0.63 & 0 \\
0 & 0 & 0.85
\end{array}\right] .
$$

The simulation results are shown in Figures 2-5. First, the bias estimation $\widehat{b}_{i}$ of the $i$ th IMU is given in Figure 2, from which it can be found that the proposed nonlinear observer is effective. The angular velocity of the $i$ th IMU is given in Figure 3.

In addition, the response curve of aircraft's angular velocity is given in Figure 4 and the response curve of aircraft's attitude is given in Figure 5.

\section{Conclusions and Future Directions}

5.1. Conclusions. The aircraft adopts a single IMU as the attitude measurement sensor, which can no longer meets the requirements of measurement accuracy. And, due to the shortage of sensors and noise, gyro will produce zero drift, which will lead to constant biases of measured angular velocity. Measurements of angular velocity with constant biases and external disturbance will make the attitude control system unstable. Therefore, the attitude anti-interference control under multiple IMUs is studied in this paper. Firstly, aiming at the zero drift, this paper adopts multiple IMUs for measurement, and the consensus observer is designed to ensure that each IMU converges to the true value of angular velocity. Aiming at the instability of aircraft control caused by external disturbances, this paper introduces a super-twisting sliding mode observer, which compensates for the external disturbances. In addition, a controller is proposed in this paper, and the actual attitude can globally asymptotically converge to the desired attitude based on the observer. Simulation results also demonstrate the effectiveness of the proposed method.

5.2. Future Directions. Future work will focus on designing observers that the zero drift biases of gyros are timevarying conditions and that the attitude information is unknown.

\section{Data Availability}

Some or all data, models, or code generated or used during the study are available from the corresponding author upon request.

\section{Conflicts of Interest}

The authors declare that there are no conflicts of interest regarding the publication of this paper.

\section{Acknowledgments}

This work was supported by the National Natural Science Foundation of China under Grant no. 52075118.

\section{References}

[1] A. Abdessameud and A. Tayebi, Motion Coordination for VTOL Unmanned Aerial Vchicles Advances in Industrial Control, Springer-Verlag, New York, NY, USA, 2013.

[2] P. Castillo, R. Lozano, and A. Dzul, Modelling and Control of Mini-Flying Machines, Springer-Verlag, New York, NY, USA, 2005.

[3] L. Wang, H. Du, W. Zhang, D. Wu, and W. Zhu, "Implementation of integral fixed-time sliding mode controller for speed regulation of PMSM servo system," Nonlinear Dynamics, vol. 102, no. 1, pp. 185-196, 2020.

[4] K. Mei and S. Ding, "Second-order sliding mode controller design subject to an upper-triangular structure," IEEE Transactions on Systems, Man, and Cybernetics: Systems, vol. 51, no. 1, p. 497, 2021.

[5] O. Mofid, S. Mobayen, and M. Khooban, "Sliding mode disturbance observer control based on adaptive synchronization in a class of fractional-order chaotic systems," International Journal of Adaptive Control and Signal Processing, vol. 33, no. 3, pp. 462-474, 2019.

[6] Q. Hou, S. Ding, and X. Yu, "Composite super-twisting sliding mode control design for PMSM speed regulation problem based on a novel disturbance observer," IEEE Transactions on Energy Conversion, p. 1, 2020.

[7] A. Norouzpour-Shirazi and F. Ayazi, "A dual-mode actuation and sensing scheme for in-run calibration of bias and scale factor errors in axisymmetric resonant gyroscopes," IEEE Sensors Journal, vol. 18, no. 5, pp. 1993-2005, 2017.

[8] M. Dai, C. Zhang, and J. Lu, "In-field calibration method for DTG IMU including g-sensitivity biases," IEEE Sensors Journal, vol. 19, no. 13, pp. 4972-4981, 2019.

[9] S. Bonnet, C. Bassompierre, C. Godin, S. Lesecq, and A. Barraud, "Calibration methods for inertial and magnetic sensors," Sensors and Actuators A: Physical, vol. 156, no. 2, pp. 302-311, 2009.

[10] R. Fontanella, D. Accardo, R. S. Lo Moriello, L. Angrisani, and D. De Simone, "MEMS gyros temperature calibration through artificial neural networks," Sensors and Actuators A: Physical, vol. 279, pp. 553-565, 2018.

[11] L. Chang, B. Hu, and K. Li, "Iterated multiplicative extended Kalman filter for attitude estimation using vector observations," IEEE Transactions on Aerospace and Electronic Systems, vol. 52, no. 4, pp. 2053-2060, 2016.

[12] P. Tsiotras, "Stabilization and optimality results for the attitude control problem," Journal of Guidance, Control, and Dynamics, vol. 19, no. 4, pp. 772-779, 1996.

[13] B. N. Stovner, T. A. Johansen, T. I. Fossen, and I. Schjølberg, "Attitude estimation by multiplicative exogenous Kalman filter," Automatica, vol. 95, pp. 347-355, 2018.

[14] H. Du, G. Wen, Y. Cheng, and J. Lu, "Design and implementation of bounded finite-time control algorithm for speed regulation of permanent magnet synchronous motor," IEEE Transactions on Industrial Electronics, vol. 68, no. 3, pp. 2417-2426, 2021.

[15] J. Li, J. Fang, and M. Du, "Error analysis and gyro-bias calibration of analytic coarse alignment for airborne POS," IEEE Transactions on Instrumentation and Measurement, vol. 61, no. 11, pp. 3058-3064, 2012.

[16] Z. Yang, D. Bi, Q. Meng, and J. Wang, "Error compensation and calibration for attitude heading reference system with four-rotor aircraft," Computer Measurement and Control, vol. 24, no. 02, pp. 267-270, 2016. 
[17] M. S. Challa, J. G. Moore, and D. J. Rogers, "A simple attitude unscented kalman filter: theory and evaluation in a magnetometer-only spacecraft scenario," IEEE Access, vol. 4, pp. 1845-1858, 2016.

[18] T. Zhang and Y. Liao, "Attitude measure system based on extended Kalman filter for multi-rotors," Computers and Electronics in Agriculture, vol. 134, pp. 19-26, 2017.

[19] R. Mahony, T. Hamel, and J.-M. Pflimlin, "Nonlinear complementary filters on the special orthogonal group," IEEE Transactions on Automatic Control, vol. 53, no. 5, pp. 1203$1218,2008$.

[20] J. Thienel and R. M. Sanner, "A coupled nonlinear spacecraft attitude controller and observer with an unknown constant gyro bias and gyro noise," IEEE Transactions on Automatic Control, vol. 48, no. 11, pp. 2011-2015, 2003.

[21] D. E. Zlotnik and J. R. Forbes, "Exponential convergence of a nonlinear attitude estimator," Automatica, vol. 72, pp. 11-18, 2016.

[22] G. Baldini, G. Steri, F. Dimc, R. Giuliani, and R. Kamnik, "Experimental identification of smartphones using fingerprints of built-in micro-electro mechanical systems (MEMS)," Sensors, vol. 16, no. 6, p. 818, 2016.

[23] Y. Al-Rawashdeh, M. Elshafei, and M. Al-Malki, "In-flight estimation of center of gravity position using all-accelerometers," Sensors, vol. 14, no. 9, pp. 17567-17585, 2014.

[24] H. Sheng and T. Zhang, "MEMS-based low-cost strap-down AHRS research," Measurement, vol. 59, pp. 63-72, 2015.

[25] M. Trkov, K. Chen, J. Yi, and T. Liu, "Inertial sensor-based slip detection in human walking," IEEE Transactions on Automation Science and Engineering, vol. 16, no. 3, pp. 1399-1411, 2019.

[26] M. Jafari, "Optimal redundant sensor configuration for accuracy increasing in space inertial navigation system," Aerospace Science and Technology, vol. 47, pp. 467-472, 2015.

[27] I. M. Brooks, "Spatially distributed measurements of platform motion for the correction of ship-based turbulent fluxes," Journal of Atmospheric and Oceanic Technology, vol. 25, no. 11, pp. 2007-2017, 2008.

[28] H. Naseri and M. R. Homaeinezhad, "Improving measurement quality of a MEMS-based gyro-free inertial navigation system," Sensors and Actuators A: Physical, vol. 207, pp. 10-19, 2014.

[29] S. Park and S. K. Hong, "Angular rate estimation using a distributed set of accelerometers," Sensors, vol. 11, no. 11, pp. 10444-10457, 2011.

[30] H. Du, C. Jiang, G. Wen, W. Zhu, and Y. Cheng, "Current sharing control for parallel DC-DC buck converters based on finite-time control technique," IEEE Transactions on Industrial Informatics, vol. 15, no. 4, pp. 2186-2198, 2019.

[31] B. Vaseghi, M. A. Pourmina, and S. Mobayen, "Finite-time chaos synchronization and its application in wireless sensor networks," Transactions of the Institute of Measurement and Control, vol. 40, no. 13, pp. 3788-3799, 2018.

[32] S. Mobayen and J. Ma, "Robust finite-time composite nonlinear feedback control for synchronization of uncertain chaotic systems with nonlinearity and time-delay," Chaos, Solitons \& Fractals, vol. 114, pp. 46-54, 2018.

[33] H. Du, G. Wen, D. Wu, Y. Cheng, and J. La, "Distributed fixed-time consensus for nonlinear heterogeneous multiagent systems," Automatica, vol. 113, pp. 1-11, 2020.

[34] J. Yuan, S. Ding, and K. Mei, "Fixed-time SOSM controller design with output constraint," Nonlinear Dynamics, vol. 102, no. 3, pp. 1567-1583, 2020.
[35] K. Mei, L. Ma, R. He, and S. Ding, "Finite-time controller design of multiple integrator nonlinear systems with input saturation," Applied Mathematics and Computation, vol. 372, Article ID 124986, 2020.

[36] F. Liu, Z. Su, H. Zhao, Q. Li, and C. Li, “Attitude measurement for high-spinning projectile with a hollow mems imu consisting of multiple accelerometers and gyros," Sensors, vol. 19, no. 8, p. 1799, 2019.

[37] L. Wang, H. Du, and D. Wu, "A coupled finite-time attitude controller and finite-time observer with an unknown constant drift bias," in Proceedings of 39th Chinese Control Conference (CCC), pp. 526-531, Kunming, China, July 2020.

[38] J. A. Moreno and M. Osorio, "Strict Lyapunov functions for the super-twisting algorithm," IEEE Transactions on Automatic Control, vol. 57, no. 4, pp. 1035-1040, 2012.

[39] R. Sepulchre, M. Jankovic, and P. Kokotovic, Constructive Nonlinear Control, Springer, London, UK, 2007.

[40] J. T.-Y. Wen and K. Kreutz-Delgado, "The attitude control problem," IEEE Transactions on Automatic Control, vol. 36, no. 10, pp. 1148-1162, 1991. 